\title{
THE CALABI FLOW WITH SMALL INITIAL ENERGY
}

\author{
Valentino Tosatti and Ben Weinkove
}

\begin{abstract}
We show that on Kähler manifolds $M$ with $c_{1}(M)=0$ the Calabi flow converges to a constant scalar curvature metric if the initial Calabi energy is sufficiently small. We prove a similar result on manifolds with $c_{1}(M)<0$ if the Kähler class is close to the canonical class.
\end{abstract}

\section{Introduction}

It has been known since Yau's proof of the Calabi conjecture that there always exists a Kähler-Einstein metric on a compact Kähler manifold $M$ with $c_{1}(M)=0[23]$ and $c_{1}(M)<0$ [23], [1]. The problem is still open in the remaining case $c_{1}(M)>0$, where existence was conjectured by Yau [24] to be equivalent to stability in the sense of geometric invariant theory. There has been much recent progress towards understanding and refining this conjecture and it is now expected, more generally, that the existence of a Kähler metric of constant scalar curvature should be equivalent to some notion of K-stability [20], [10].

A natural approach to the problem of existence of these canonical metrics is to consider the Calabi flow. Fix a Kähler metric $\omega=\frac{\sqrt{-1}}{2} g_{i \bar{j}} d z^{i} \wedge d z^{\bar{j}}$. Then the Calabi flow is a flow of Kähler potentials $\varphi=\varphi(t)$ given by

$$
\frac{\partial}{\partial t} \varphi=R\left(\omega_{\varphi}\right)-\mu
$$

where $\omega_{\varphi}=\omega+\frac{\sqrt{-1}}{2} \partial \bar{\partial} \varphi>0, R\left(\omega_{\varphi}\right)$ is the scalar curvature of the metric $\omega_{\varphi}$, and

$$
\mu=\frac{\pi n\left[c_{1}(M)\right] \cdot[\omega]^{n-1}}{[\omega]^{n}}, \quad n=\operatorname{dim}_{\mathbb{C}} M .
$$

It is a gradient flow for the Mabuchi energy [14], and it decreases the Calabi functional, which we define by

$$
\mathrm{Ca}\left(\omega_{\varphi}\right)=\int_{M}\left(R\left(\omega_{\varphi}\right)-\mu\right)^{2} \omega_{\varphi}^{n}
$$

(this differs from the usual definition by a constant). It is expected that the Calabi flow should converge to a constant scalar curvature ( $\mathrm{cscK}$ ) metric when one exists [11]. Unfortunately, since the flow is a parabolic fourth order equation, there are very few techniques available to tackle this kind of analytic question. In the case of

Received by the editors October 8, 2006.

Most of this work was carried out while the second author was visiting Imperial College on a Royal Society Research Assistantship; the second author is also supported in part by National Science Foundation grant DMS-05-04285; the first author is supported by a Cooper-Siegel Graduate Student Fellowship Fund. 
Riemann surfaces, Chruściel [9] showed that the flow always converges to a constant curvature metric, making use of the existence of such a metric and the Bondi mass of general relativity. Chen [7] (see also [19]) gave a different proof of Chruściel's theorem using concentration-compactness results. The analytic difficulties become more acute in higher dimensions, but some progress has been made [4], [8]. In particular, Chen and He [8] recently showed that the Calabi flow exists as long as the Ricci curvature remains bounded, and proved a short time existence result. They also showed that if the initial metric is close to a cscK metric in the $C^{3+\alpha}$ sense, for some $\alpha>0$, then the flow will converge to a cscK metric.

In this paper we restrict to manifolds satisfying $c_{1}(M)=0$ and $c_{1}(M)<0$ and consider the case when the initial data has small Calabi energy, in a suitable sense. When $c_{1}(M)=0$ every class admits a unique Kähler Ricci-flat metric by Yau's theorem. We have the following result.

Theorem 1. Let $M$ be a compact Kähler manifold with $c_{1}(M)=0$. Fix constants $K_{1}, K_{2}$ and a reference Kähler metric $\omega$. Then there exists $\varepsilon>0$ depending only on $\omega, K_{1}, K_{2}$ such that if $\omega_{0}$ is any Kähler metric in $[\omega]$ satisfying:

(i) $-K_{1} \omega \leq \operatorname{Ric}\left(\omega_{0}\right) \leq K_{2} \omega$

(ii) $\mathrm{Ca}\left(\omega_{0}\right)<\varepsilon$,

then the solution of the Calabi flow starting at $\omega_{0}$ exists for all time and converges in $C^{\infty}$ to the Kähler Ricci-flat metric in $[\omega]$.

In the case $c_{1}(M)<0$ we know that there exists a Kähler-Einstein metric in the class $-\pi c_{1}(M)$, and, by the implicit function theorem, there exist cscK metrics in nearby classes [13]. Indeed, it is expected by the results of [5], [22], [18] that there exist cscK metrics in a larger neighborhood of $-\pi c_{1}(M)$ (but not necessarily in every class $[15])$. We show that the Calabi flow converges to the unique cscK metric if the Calabi energy is small and if the class is sufficiently close to $-\pi c_{1}(M)$.

Fix a norm $\|\cdot\|_{N}$ on the vector space $H_{\mathbb{R}}^{1,1}(M)$. Then we have the following:

Theorem 2. Let $M$ be a compact Kähler manifold with $c_{1}(M)<0$. Fix two constants $K_{1}, K_{2}$ and a reference metric $\omega \in-\pi c_{1}(M)$ with $\operatorname{Ric}(\omega)<0$. Then there exist constants $\varepsilon, \delta>0$ depending only on $\omega, K_{1}, K_{2}$ and $\|\cdot\|_{N}$ such that if a Kähler metric $\omega_{0}$ satisfies the conditions:

(i) $\left\|\left[\omega_{0}\right]-[\omega]\right\|_{N}<\delta$

(ii) $R\left(\omega_{0}\right) \geq-K_{1}$

(iii) $\operatorname{Ric}\left(\omega_{0}\right) \leq K_{2} \omega$

(iv) $\mathrm{Ca}\left(\omega_{0}\right)<\varepsilon$,

then the solution of the Calabi flow starting at $\omega_{0}$ exists for all time and converges in $C^{\infty}$ to a cscK metric in $\left[\omega_{0}\right]$.

Note that we do not assume a priori that a cscK metric exists in the given class. The results here are quite different from the stability result of [8] in these cases, where it is assumed that a cscK metric exists and that the initial metric is close to the $\operatorname{cscK}$ metric in the $C^{3+\alpha}$ norm. 
In section 2, we prove two key compactness lemmas for Kähler metrics, which rely on Yau's estimates. These, along with the 'short-time existence estimates' of Chen-He and the fact that the Calabi functional is decreasing, are used in section 3 to prove Theorems 1 and 2. In the course of the proofs, we will use the letter $C$ to denote a suitably uniform positive constant, which may differ from line to line.

\section{Compactness results}

In this section we prove two compactness results for Kähler metrics. The first is as follows.

Lemma 1. Let $M$ be a compact Kähler manifold. Fix a reference Kähler metric $\omega$ and suppose $\omega^{\prime}=\omega+\frac{\sqrt{-1}}{2} \partial \bar{\partial} \varphi$ is a metric satisfying

$$
-K_{1} \omega \leq \operatorname{Ric}\left(\omega^{\prime}\right) \leq K_{2} \omega .
$$

Then for any $0<\alpha<1$ there exist positive constants $c$ and $A$ depending only on $\omega$, $\alpha, K_{1}$ and $K_{2}$ such that $\omega^{\prime} \geq c \omega$ and

$$
\|\varphi-\underline{\varphi}\|_{C^{3+\alpha}(\omega)} \leq A
$$

where $\underline{\varphi}=\frac{1}{V} \int_{M} \varphi \omega^{n}$, for $V=\int_{M} \omega^{n}$.

Proof. Define

$$
F:=\log \frac{\omega^{\prime n}}{\omega^{n}}
$$

Calculate

$$
\Delta F=R(\omega)-g^{i \bar{\jmath}} R_{i \bar{\jmath}}^{\prime},
$$

where we are using the standard notation $R_{i \bar{\jmath}}^{\prime}$ for the Ricci curvature of the metric $\omega^{\prime}$.

Our assumptions imply $|\Delta F| \leq C$. Applying Green's formula for the metric $\omega$ we see that for all $x \in M$

$$
F(x)=\frac{1}{V} \int_{M} F \omega^{n}-\int_{y \in M} \Delta F(y) G(x, y) \omega^{n}(y),
$$

where the Green's function $G$ is bounded in $L^{1}(\omega)$ and uniformly from below. Hence

$$
\sup _{M}|F| \leq\left|\frac{1}{V} \int_{M} F \omega^{n}\right|+C .
$$

It remains then to bound the integral of $F$. But from the definition of $F$ there must exist some point $x_{0} \in M$ with $F\left(x_{0}\right)=0$ and so applying (3) with $x=x_{0}$ shows that $\int_{M} F \omega^{n}$ is bounded above and below. (In fact, by Jensen's inequality and the concavity of the log function it is easy to see that we can take the upper bound to be zero.)

Now that we have $|F| \leq C$ and $\Delta F \geq-C$, Yau's second order estimates [23, Prop. 2.1] give us bounds

$$
\sup _{M}|\varphi-\underline{\varphi}| \leq C, \quad C^{-1} \omega \leq \omega^{\prime} \leq C \omega
$$

We also notice that $|\Delta F| \leq C$ together with the elliptic estimates for $\Delta$ give uniform $L_{2}^{p}(\omega)$ bounds on $F$ for all $p \geq 1$. We can now use the method of Evans and Krylov (see 
also the simplification by Trudinger [21], and the exposition of Siu [17]) that employs Moser's Harnack inequality to obtain the Hölder estimate $\|\varphi-\varphi\|_{C^{2+\beta}(\omega)} \leq C$ for some uniform $\beta>0$. Let $\psi$ be a local potential for $\omega$ so that $\omega^{\prime}=\frac{\sqrt{-1}}{2} \partial \bar{\partial}(\varphi+\psi)$ locally. Differentiate the equation (1) with respect to $z^{i}$ to obtain

$$
\frac{\partial F}{\partial z^{i}}=\Delta^{\prime} \frac{\partial(\varphi+\psi)}{\partial z^{i}}-\frac{\partial}{\partial z^{i}} \log \operatorname{det} g .
$$

The operator $\Delta^{\prime}$ is uniformly elliptic with coefficients bounded in $C^{\beta}(\omega)$ and $F$ has uniform $L_{2}^{p}(\omega)$ bounds for all $p \geq 1$, so the elliptic estimates give uniform $L_{4}^{p}(\omega)$ bounds on $\varphi-\varphi$ and Sobolev's embedding theorem gives uniform $C^{3+\alpha}(\omega)$ bounds after taking $p$ sufficiently large. Q.E.D.

For the case $c_{1}(M)<0$ we will use instead the following compactness result.

Lemma 2. Let $M$ be a compact Kähler manifold with $c_{1}(M)<0$. Fix a reference Kähler metric $\omega$ with $\operatorname{Ric}(\omega)<0$ and suppose $\omega^{\prime}=\omega+\frac{\sqrt{-1}}{2} \partial \bar{\partial} \varphi$ is a metric satisfying

$$
R\left(\omega^{\prime}\right) \geq-K_{1} \quad \text { and } \quad \operatorname{Ric}\left(\omega^{\prime}\right) \leq K_{2} \omega .
$$

Then for any $0<\alpha<1$ there exist positive constants $c$ and $A$ depending only on $\omega$, $\alpha, K_{1}$ and $K_{2}$ such that $\omega^{\prime} \geq c \omega$ and

$$
\|\varphi-\underline{\varphi}\|_{C^{3+\alpha}(\omega)} \leq A .
$$

Proof. Using the same notation as in the proof of Lemma 1 we see immediately that $\Delta F \geq-C$. It then follows from (3) that $\sup _{M} F \leq C$. Calculate

$$
\Delta^{\prime} F=g^{i \bar{\jmath}} R_{i \bar{\jmath}}-R\left(\omega^{\prime}\right) .
$$

By assumption we know that $\operatorname{Ric}(\omega) \leq-C^{-1} \omega$. To obtain the lower bound of $F$ we use the following argument from [16]. Consider a point $x$ where $F$ attains its minimum. At $x$,

$$
0 \leq \Delta^{\prime} F \leq-C^{-1} g^{i \bar{\jmath}} g_{i \bar{\jmath}}+K_{1},
$$

and so $g^{\prime i \bar{j}} g_{i \bar{\jmath}}(x) \leq C$. But the geometric-harmonic means inequality implies that at $x$,

$$
e^{F}=\frac{\omega^{\prime n}}{\omega^{n}} \geq\left(\frac{n}{g^{\prime i \bar{\jmath}} g_{i \bar{\jmath}}}\right)^{n} \geq C
$$

and so $\sup _{M}|F| \leq C$. Then from Yau's estimates, as above, we see that $\omega$ and $\omega^{\prime}$ are uniformly equivalent. Since $\operatorname{Ric}\left(\omega^{\prime}\right) \leq K_{2} \omega$ we see that $\partial_{i} \partial_{\bar{\jmath}} F \geq R_{i \bar{\jmath}}-K_{2} g_{i \bar{\jmath}}$ and it follows that

$$
\Delta F \leq C \Delta^{\prime} F+\tilde{C},
$$

for a uniform constant $\tilde{C}$. From (4), this is uniformly bounded from above. The rest of the argument follows as in Lemma 1. Q.E.D. 


\section{The Calabi flow}

In this section we prove the main theorems. We first show that, under the assumptions of Theorems 1 and 2, the Calabi flow exists for all time and the evolving metric and its derivatives are uniformly bounded. In order to make use of the Calabi functional, which decreases along the flow, we require the following well-known formula [3], which holds for any Kähler metric $\omega$ :

$$
\mathrm{Ca}(\omega)=\int_{M}\left|\operatorname{Ric}(\omega)-\frac{\mu}{n} \omega\right|^{2} \omega^{n}+\Psi,
$$

where

$$
\Psi=n(n-1) \pi^{2}\left(\left[c_{1}(M)\right]^{2} \cdot[\omega]^{n-2}-\frac{\left(\left[c_{1}(M)\right] \cdot[\omega]^{n-1}\right)^{2}}{[\omega]^{n}}\right) .
$$

Remark. [12, Corollary 1.6.3] Assume that $\pm c_{1}(M)>0$. Then it is interesting to note that $\Psi \leq 0$ with equality if and only if $[\omega]$ is a multiple of $c_{1}(M)$. However, this inequality has the wrong sign to be useful to us here.

We assume the hypotheses of Theorem 1 , where $\varepsilon$ is to be determined later. Let $\omega_{t}=\omega+\frac{\sqrt{-1}}{2} \partial \bar{\partial} \varphi_{t}$ be the solution of the Calabi flow starting at $\omega_{0}$. Applying Lemma 1 to $\omega_{0}$ we immediately see that $\left\|\varphi_{0}-\varphi_{0}\right\|_{C^{3+\alpha}(\omega)} \leq C$ and $\omega_{0} \geq C^{-1} \omega$ for any fixed $0<\alpha<1$. By the short time existence result of [8], the Calabi flow starting at $\omega_{0}$ exists on $\left[0, t_{0}\right]$ where $t_{0}>0$ is uniform, and on the subinterval $\left[t_{0} / 2, t_{0}\right]$ we have uniform $C^{k+\alpha}(\omega)$ bounds on $\varphi_{t}$ for all $k \geq 4$. In particular there exist uniform constants $K_{3}, K_{4}$ such that for $t \in\left[t_{0} / 2, t_{0}\right]$ we have

$$
-K_{3} \omega \leq \operatorname{Ric}\left(\omega_{t}\right) \leq K_{4} \omega \text {. }
$$

Now consider the set

$$
\mathcal{T}:=\left\{\begin{array}{l|l}
t \geq t_{0} / 2 & \begin{array}{l}
\text { the flow exists on the time interval }[0, t] \text { and } \\
-2 K_{3} \omega \leq \operatorname{Ric}\left(\omega_{t^{\prime}}\right) \leq 2 K_{4} \omega \text { for } t^{\prime} \in\left[t_{0} / 2, t\right]
\end{array}
\end{array}\right\} .
$$

From what we have just shown, there exist uniform $C^{k}(\omega)$ bounds along the flow for all $k$ as long as $t$ remains in the set $\mathcal{T}$.

Suppose that $\tau=\sup \mathcal{T}<\infty$. We will show that there exists a uniform $\varepsilon$ such that $\mathrm{Ca}\left(\omega_{\tau}\right)>\varepsilon$, thus contradicting assumption (ii). Since $\tau \in \mathcal{T}$, we have uniform bounds for the metric $\omega_{\tau}$ and its derivatives. At time $\tau$ there exists a point $x \in M$ at which the following happens. Diagonalizing $\operatorname{Ric}\left(\omega_{\tau}\right)$ with respect to $\omega$, one of the eigenvalues of $\operatorname{Ric}\left(\omega_{\tau}\right)$ must equal $2 K_{4}$ or $-2 K_{3}$. But $\omega_{\tau}$ and $\omega$ are uniformly equivalent and we have uniform estimates on $\omega_{\tau}$ and its derivatives, so there exists a uniform $\varepsilon>0$ such that

$$
\int_{M}\left|\operatorname{Ric}\left(\omega_{\tau}\right)\right|^{2} \omega_{\tau}^{n} \geq \varepsilon,
$$

and we obtain a contradiction from (5) since $\Psi=0$.

We turn now to the case of Theorem 2. First, if $\delta>0$ is sufficiently small then there exists a reference metric $\tilde{\omega}$ in $\left[\omega_{0}\right]$, uniformly equivalent to the original $\omega$, with 
uniformly bounded derivatives and $\operatorname{Ric}(\tilde{\omega})<-C^{-1} \tilde{\omega}$. Set $\omega_{t}=\tilde{\omega}+\frac{\sqrt{-1}}{2} \partial \bar{\partial} \varphi_{t}$. From the assumptions (ii) and (iii) we can apply Lemma 2 to obtain a uniform $C^{3+\alpha}(\tilde{\omega})$ bound on $\varphi_{0}-\varphi_{0}$. We can then use a very similar argument to the one above. We replace (6) by

$$
R\left(\omega_{t}\right) \geq-K_{3}, \operatorname{Ric}\left(\omega_{t}\right) \leq K_{4} \tilde{\omega}
$$

and replace $\mathcal{T}$ by

$$
\mathcal{T}:=\left\{\begin{array}{l|l}
t \geq t_{0} / 2 \mid \begin{array}{l}
\text { the flow exists on the time interval }[0, t] \text { and } \\
R\left(\omega_{t^{\prime}}\right) \geq-2 K_{3}, \operatorname{Ric}\left(\omega_{t^{\prime}}\right) \leq 2 K_{4} \tilde{\omega} \text { for } t^{\prime} \in\left[t_{0} / 2, t\right]
\end{array}
\end{array}\right\} .
$$

If $\tau=\sup \mathcal{T}<\infty$, then at some point $x$ in $M$ either $R\left(\omega_{\tau}\right)=-2 K_{3}$ or one of the eigenvalues of $\operatorname{Ric}\left(\omega_{t}\right)$ with respect to $\tilde{\omega}$ is equal to $2 K_{4}$. In the first alternative, since $-2 K_{3}<\mu$, there exists a uniform $\varepsilon$ such that $\mathrm{Ca}\left(\omega_{\tau}\right)>\varepsilon$. Otherwise, there exists $\varepsilon>0$ such that

$$
\int_{M}\left|\operatorname{Ric}\left(\omega_{\tau}\right)-\frac{\mu}{n} \omega_{\tau}\right|^{2} \omega_{\tau}^{n}>2 \varepsilon
$$

(using the fact that $\mu<0$ ). Picking $\delta$ small enough so that $\Psi>-\varepsilon$ we obtain a contradiction in the second alternative.

To complete the proofs of Theorems 1 and 2, it remains to prove convergence of the flow. Since we have uniform $C^{k}$ bounds along the flow, we know that for every sequence of times $t_{k} \rightarrow \infty$ there exists a subsequence $t_{k_{j}}$ and a metric $\omega_{\infty}$ such that $\omega_{t_{k_{j}}}$ converges in $C^{\infty}$ to $\omega_{\infty}$ as $j \rightarrow \infty$. It is shown in [8] that such a limit $\omega_{\infty}$ must be an extremal metric (that is, a critical metric of Calabi's functional). But extremal metrics are unique in their Kähler classes in the cases $c_{1}(M)=0[2]$ and $c_{1}(M)<0$ [2], [6]. It follows that the Calabi flow converges in $C^{\infty}$ to $\omega_{\infty}$, where $\omega_{\infty}$ is the unique Kähler Ricci-flat or cscK metric in $\left[\omega_{0}\right]$. Q.E.D.

\section{Acknowledgements}

The authors would like to thank Professor S.-T. Yau, the first author's thesis advisor, for a number of very helpful discussions. The authors are also grateful to Professor D.H. Phong for his support and advice and to Professor J. Sturm for some useful comments on an earlier version of this paper.

\section{References}

[1] T. Aubin, Équations du type Monge-Ampère sur les variétés kählériennes compactes, Bull. Sci. Math. 102 (1978), no. 1, 63-95.

[2] E. Calabi, The space of Kähler metrics, In Proceedings of the International Congress of Mathematicians, Amsterdam, 1954, Vol. 2, 206-207, North-Holland, Amsterdam, 1956.

[3] _ Extremal Kähler metrics, in Ann. of Math. Stud. 102, Princeton Univ. Press, 1982, 259-290.

[4] S. C. Chang, Recent developments on the Calabi flow, Geometric evolution equations, 17-42, Contemp. Math. 367, Amer. Math. Soc., Providence, RI, 2005.

[5] X. X. Chen, On the lower bound of the Mabuchi energy and its application, Internat. Math. Res. Notices 2000, no. 12, 607-623.

[6] _ , The space of Kähler metrics, J. Differential Geom. 56 (2000), no. 2, 189-234.

[7] Calabi flow in Riemann surfaces revisited: a new point of view, Internat. Math. Res. Notices 2001, no. 6, 275-297. 
[8] X. X. Chen and W. Y. He, On the Calabi flow, preprint arXiv math.DG/0603523.

[9] P. T. Chruściel, Semi-global existence and convergence of solutions of the Robinson-Trautman (2-dimensional Calabi) equation, Comm. Math. Phys. 137 (1991), no. 2, 289-313.

[10] S. K. Donaldson, Scalar curvature and stability of toric varieties, J. Differential Geom. 62 (2002), no. 2, 289-349.

[11] _ Conjectures in Kähler geometry, in Strings and geometry, 71-78, Clay Math. Proc. 3, AMS 2004

[12] R. Lazarsfeld, Positivity in Algebraic Geometry I, Springer-Verlag, 2004.

[13] C. LeBrun and S. R. Simanca, Extremal Kähler metrics and complex deformation theory, Geom. Funct. Anal. 4 (1994), no. 3, 298-336.

[14] T. Mabuchi, K-energy maps integrating Futaki invariants, Tôhoku Math. Journ. 38 (1986), $575-593$.

[15] J. Ross, Unstable products of smooth curves, Invent. Math. 165 (2006), no. 1, 153-162.

[16] W. D. Ruan, On the convergence and collapsing of Kähler metrics, J. Differential Geom. 52 (1999), 1-40.

[17] Y. T. Siu, Lectures on Hermitian-Einstein metrics for stable bundles and Kähler-Einstein metrics, Birkhäuser Verlag, 1987.

[18] J. Song and B. Weinkove, On the convergence and singularities of the J-flow with applications to the Mabuchi energy, preprint arXiv math.DG/0505476.

[19] M. Struwe, Curvature flows on surfaces, Ann. Sc. Norm. Super. Pisa Cl. Sci. 1 (2002), no. 2, $247-274$.

[20] G. Tian, Kähler-Einstein metrics with positive scalar curvature, Invent. Math. 137 (1997), $1-37$.

[21] N. S. Trudinger, Local estimates for subsolutions and supersolutions of general second order elliptic quasilinear equations, Invent. Math. 61 (1980), no. 1, 67-79.

[22] B. Weinkove, The J-flow in higher dimensions and the lower boundedness of the Mabuchi energy, J. Diff. Geom. 73 (2006), no. 2, 351-358.

[23] S. T. Yau, On the Ricci curvature of a compact Kähler manifold and the complex Monge-Ampère equation, I, Comm. Pure Appl. Math. 31 (1978), 339-411.

[24] _ Open problems in geometry, Proc. Sympos. Pure Math. 54 (1993), 1-28 (problem 65).

Department of Mathematics, Harvard University, Cambridge, MA 02138

E-mail address: tosatti@math.harvard.edu, weinkove@math.harvard.edu 